\begin{abstract}
Aus der Bayerischen Landesanstalt für Tierzucht, Grub' und der Lehr- und Versuchsanstalt für Tierhaltung, Schwarzenau

WERNER WITTMANN' ${ }^{\prime}$, KAY-UWE GÖTZ', WALTER PESCHKE', JOHANN-PETER LINDNER ${ }^{2}$ und MAXIMILIAN HAUSER ${ }^{1}$

\section{Einfluß des MHS-Genotyps auf die Mast- und Schlachtleistung von Piétrainschweinen und PI x DL-Mastendprodukten in der Stationsprufung*}

\begin{abstract}
Summary
Title of the paper: The influence of MHS-genotypes on fattening and carcass performance traits of purebred Piétrain pigs and PI x DL slaughter pigs at testing station

For the comparison of fattening and carcass performance traits as well as the meat quality of different MHS. genotypes, one Piétrain group with 434 and another one of 64 PI x DL animals of both sexes were evaluated. The LSQ-means from an animal model showed in tendency a lower fattening performance of the stresssusceptible genotypes. Significant differences were found for higher fat layers and fat surfaces and for lower meat surfaces and percentage of lean meat in NP- and NN-genotypes as compared to the PP-animals. The differences between the MHS-genotypes of the PI x DL animals were similar to those of the Piétrain animals. Both statistical models confirmed a better meat quality already in the NP-genotypes. With respect to lower loss rates, reduction of insufficient meat qualities (PSE, DFD) and a still high proportion of lean meat of the NP- and $\mathrm{NN}$ - animals, a selection of the Piétrain race for stress-resistance should be suitable.
\end{abstract}

Key words: Piétrain, MHS-genotype, fattening and carcass performance, meat quality

\title{
Zusammenfassung
}

An zwei Stichproben wurde die Auswirkung des MHS-Genotyps auf die Mast- und Schlachtleistung sowie die Fleischbeschaffenheit untersucht. Dafür standen zwei Tiergruppen aus der Stationsprüfung zur Verfügung. Eine Gruppe umfaßte 434 weibliche Piétrain Reinzuchttiere, die andere mit 64 PI x DL Kreuzungstieren stammte aus Ferkelerzeugerbetrieben, in denen MHS-misch- und reinerbig negative KB-Eber eingesetzt worden waren. Gesicherte Unterschiede ergaben sich in der Erhöhung der Speckmaße und Fettfläche, sowie der Abnahme der Fleischfläche und des Fleischanteils bei den NP- und NN-Genotypen zu den PP-Genotypen. Die Differenzen der MHS-Genotypen der PI x DL-Kreuzungen lagen nach dem Tiermodell auf dem Niveau der Piétraintiere. Die Fleischbeschaffenheit verbesserte sich bereits erheblich in den NP-Genotypen nach beiden Auswertungsmodellen. Bei der Mastleistung hatten die PP-Reinzuchttiere schlechtere Ergebnisse, während sich bei den Kreuzungen eine umgekehrte Tendenz zeigte. Die geringeren Verluste, das reduzierte Auftreten von Fleischbeschaffenheitsmångeln (PSE, DFD) bei einem hohen Fleischanteil der NP- und NN- Reinzucht- und Kreuzungstiere lassen eine Streßsanienung der Vaterrasse Piétrain sinnvoll erscheinen.

Schlüsselwörter: Piétrain, MHS-Genotypen, Mast- und Schlachtleistung, Fleischbeschaffenheit

\section{Einleitung}

Der MHS-Gentest ist seit dem 1.1.1992 in der bayerischen Herdbuchzucht als Hilfsmittel zum Aufbau streßstabiler DL-Sauenlinien eingeführt. Nach FÖRSTER et al.

\footnotetext{
- Der Arbeitsgemeinschaf nordbayerischer Schweineproduzenten sei fur die Forderung der Untersuchung freundlichst gedankt.
} 
(1992) ermöglicht dieses Verfahren zur Feststellung der Streßstabilität die Differenzierung der Tiere nach den Genotypen NN (reinerbig streßunempfindlich), NP (mischerbig streßunempfindlich) und PP (reinerbig streßempfindlich) als Eigenleistungsprüfung. Die bisherigen Untersuchungen von WITTMANN et al. (1992 und 1993) an homozygot streßresistenten DL-Sauen und DL-Kastraten aus der Stationsprüfung (LPA) ergaben Veränderungen in verschiedenen Leistungsmerkmalen. Hierbei zeigten reinerbig streßstabile Tiere, neben einer Reduzierung des Fleischanteiles von bis zu $1,5 \%$ und der Fleischfläche von bis $\mathrm{zu} 3,2 \mathrm{~cm}^{2}$, ein verschlechtertes Fleisch:FettVerhältnis und eine Erhöhung der Speckmaße bei einer deutlichen Verbesserung der Fleischbeschaffenheitsmerkmale.

Das erste Auftreten mischerbig streßresistenter Piétraintiere führte zu Überlegungen zum Aufbau reinerbiger streßresistenter Piétrainlinien (BIEDERMANN et al., 1997).

Durch diese Situation in der Zucht, sowie die sich verändernden Kostenstrukturen in der Produktion und die gestiegenen Qualitätsanforderungen der Abnehmer, erhebt sich die Frage, mit welchen Leistungsänderungen bei einer MHS-Sanierung der Rasse Piétrain zu rechnen ist (GLODEK, 1996; THOLEN, 1996) und wie sich diese auf die Erzeugung von Mastendprodukten der Einfachkreuzung PI x DL auswirkt.

\section{Material und Methode}

Zur Beantwortung dieser Fragestellungen standen zwei Tiergruppen für die Auswertungen zur Verfügung. Die erste Gruppe aus der Piétrain Reinzucht bestand aus 434 zur Nachkommenprüfung an der LPA Schwarzenau eingestallten weiblichen Tieren. Insgesamt stammten sie von 110 Ebern und 248 Sauen ab (Tab. 1). Bezogen auf die MHS-Untergruppen wurden 150 Eber und 286 Sauen eingesetzt. Die Versuchstiere wurden nach den LPA-Richtlinien auf Mast-, Schlachtleistung und Fleischbeschaffenheit geprüft und bei einem Lebendgewicht von ca. $104 \mathrm{~kg}$ geschlachtet, um ein Schlachtkörpergewicht von $85 \mathrm{~kg}$ zu erreichen. Entweder wurde bei der Einstallung, oder nach der Tötung bei der Schlachtung, mittels Kerbzange eine Gewebeprobe aus dem Ohr zur MHS-Genotypisierung nach dem Münchner MHS-Gentest (DOVC, 1992) entnommen.

\section{Tabelle 1}

Verteilung der eingestallten weiblichen PI-HB-Tiere nach MHS-Genstatus, Väter und Mütter $(n=434)$ (Distribution of the female PI-HB-animals by MHS-gene status, boars and sows)

\begin{tabular}{cccc}
\hline $\begin{array}{c}\text { MHS-Status } \\
\text { der Nachkommen }\end{array}$ & Tiere & Väter & Mütter \\
\hline PP & 257 & 81 & 162 \\
NP & 111 & 48 & 45 \\
NN & 66 & 21 & 79 \\
\hline Tiere gesamt & 434 & 150 & 286 \\
\hline
\end{tabular}

Die Auswertung erfolgte mit einem BLUP-Tiermodell unter Berücksichtigung der additiv genetischen Verwandschaft. Das lineare Modell für das Programm PEST 3.1 (GROENEVELD, 1993) verwendete die von SCHMUTZ (1995) geschätzten genetischen Parameter. Das Modell lautete: 


$$
\mathrm{y}=\mu+\text { MHS + MPJQUART + GRUPPE + TIER + e }
$$

$\begin{array}{lll}\mathrm{y} & = & \text { Beobachtungswert } \\ \mu & = & \text { Mittelwert } \\ \text { MHS } & = & \text { fixer Effekt des MHS-Genotyps } \\ \text { MPJQUART } & = & \text { fixer Effekt der Prüfungsanstalt-Jahr-Quartalsklasse } \\ \text { GRUPPE } & = & \text { zufälliger Effekt der genetischen Wurfumwelt } \\ \text { TIER } & = & \text { zufälliger Effekt des Tieres } \\ \mathrm{e} & = & \text { zufälliger Resteffekt }\end{array}$

Bei den Schlachtleistungsmerkmalen wurde zusätzlich die Regression auf das Schlachtgewicht warm berücksichtigt.

Für die zweite Versuchsgruppe der 64 PI x DL-Mastendprodukte waren 4 KB-PI-Eber des Genotyps NP an 16 Ferkelerzeuger DL-Sauen angepaart worden, mit maximal 14 Tagen Differenz der Besamungstermine. Die Nachkommen aus insgesamt 9 Würfen verteilten sich auf zwei Geschlechter mit NN- oder NP-MHS-Genstatus, d.h. 16 Tiere je Untergruppe, und wurden nach den LPA-Richtlinien in Vierergruppen an der LPA Grub geprüft. Die Entnahme der Gewebeproben und die untersuchten Leistungsmerkmale entsprachen dem Vorgehen wie bei der Piétrain-Gruppe. Die varianzanalytischen Auswertungen berücksichtigten neben dem fixen Effekt des MHS-Genstatus noch das Geschlecht und die Interaktion MHS x Geschlecht. Auch hier wurde bei den Schlachtleistungsmerkmalen die Regression auf das Schlachtgewicht warm berücksichtigt. Bei den 58 PI x DL-Tieren mit auswertbaren Schlachtleistungsdaten konnten analog zu den PI-HB-Tieren der ersten Versuchsgruppe mit einem Tiermodell eine zusätzliche Auswertung vorgenommen werden:

\begin{tabular}{lll}
\multicolumn{3}{c}{$\mathrm{y}=\mu+$ MHS + SEX + MHS x SEX + WURF + TIER + e } \\
$y$ & $=$ & Beobachtungswert \\
$\mu$ & $=$ & Mittelwert \\
MHS & $=$ & fixer Effekt des MHS-Genotyps \\
SEX & $=$ & Interaktion MHS-Status x Geschlecht \\
MHS x SEX & $=$ & zufälliger Effekt der Wurfumwelt \\
WURF & $=$ & zufälliger Effekt des Tieres \\
TIER & $=$ & zufälliger Resteffekt
\end{tabular}

Für die Genotypen PP und NP wurden für beide Gruppen noch die Verteilung des Auftretens der Fleischbeschaffenheitsmängel PSE und DFD durch Klassenbildung der pH-Werte im Schinken und Kotelett berechnet.

3 Ergebnisse und Diskussion

Weibliche Piétrain Reinzuchttiere

Von 434 eingestallten weiblichen PI-HB-Tiere entsprachen 257 (59,2\%) dem MHSGenotyp PP, 111 (25,6 \%) dem Typ NP und 66 (15,2\%) waren homozygot negativ NN (Tab. 1). 
Die verschiedenen Leistungsmerkmale nach MHS-Genotyp zeigt die Tabelle 2. Demnach haben PP Tiere eine um bis zu $32 \mathrm{~g}$ schlechtere Zunahme im Prüfungsabschnitt als NP- oder NN-Tiere $(\mathrm{P}<0.004)$, im Gegensatz zu früheren Untersuchungen von PESCHKE et al. (1993).

Tabelle 2

LSQ-Mittelwerte der weiblichen PI-HB-Tiere nach MHS-Genotypen $(n=323)$ (LSQ-means of the female PIHB-animals by MHS-genotypes)

\begin{tabular}{|c|c|c|c|c|}
\hline \multirow[b]{2}{*}{ Merkmal } & \multicolumn{3}{|c|}{ LSQ-Mittelwert } & \multirow[b]{2}{*}{$\begin{array}{c}\text { p-Wert } \\
\text { MHS }\end{array}$} \\
\hline & $\begin{array}{c}\text { PP } \\
\mathrm{n}=257\end{array}$ & $\begin{array}{c}\mathrm{NP} \\
\mathrm{n}=111\end{array}$ & $\begin{array}{c}\mathrm{NN} \\
\mathrm{n}=66\end{array}$ & \\
\hline Zunahme i. Abschnitt & $656 \mathrm{a}$ & $688 \mathrm{~b}$ & $677 \mathrm{~b}$ & $0,004^{* * *}$ \\
\hline Schlachtkörperlänge & $92,2 \mathrm{a}$ & $93,8 \mathrm{~b}$ & $94,4 \mathrm{c}$ & $0,000^{* * *}$ \\
\hline Rückenspeckdicke & $1,62 \mathrm{a}$ & $1,75 \mathrm{~b}$ & $1,77 \mathrm{~b}$ & $0,001 *$ \\
\hline Seitenspeckdicke & $1,25 \mathrm{a}$ & $1,50 \mathrm{~b}$ & $1,57 \mathrm{~b}$ & $0,000^{* * *}$ \\
\hline Speckmaß B & $0,58 \mathrm{a}$ & $0,74 \mathrm{~b}$ & $0,78 \mathrm{c}$ & $0,000^{* *}$ \\
\hline Fettfläche & $10,4 \mathrm{a}$ & $11,5 \mathrm{~b}$ & $14,5 \mathrm{c}$ & $0,000^{* *}$ \\
\hline Fleischfläche & $65,0 \mathrm{a}$ & $61,0 \mathrm{~b}$ & $59,3 \mathrm{c}$ & $0,000^{* *}$ \\
\hline Fleisch:Fett-Verhältnis & 0,17 a & $0,21 \mathrm{~b}$ & $0,23 \mathrm{~b}$ & $0,000^{* *}$ \\
\hline Fleischanteil (LPA) & $66,6 \mathrm{a}$ & $64,6 \mathrm{~b}$ & $63,9 \mathrm{~b}$ & $0,000^{* *}$ \\
\hline Schinkenanteil & $35,3 \mathbf{a}$ & $34,5 \mathrm{~b}$ & $34,4 \mathrm{~b}$ & $0,000^{* *}$ \\
\hline $\mathrm{pH}_{1}$-Kotelett & $5,70 a$ & $6,16 \mathrm{~b}$ & $6,35 \mathrm{c}$ & $0,000^{\text {*** }}$ \\
\hline $\mathrm{pH}_{24}$-Kotelett & $5,46 \mathrm{a}$ & 5,47 a & $5,53 \mathrm{~b}$ & $0,009^{* *}$ \\
\hline $\mathrm{pH}_{1}-$ Schinken & $5,87 \mathrm{a}$ & $6,28 \mathrm{~b}$ & $6,58 \mathrm{c}$ & $0,000^{* * *}$ \\
\hline $\mathrm{pH}_{24}$-Schinken & 5,59 & 5,59 & 5,61 & 0,657 \\
\hline Fleischhelligkeit & $64,0 \mathrm{a}$ & $68,1 \mathrm{~b}$ & $68,3 \mathrm{~b}$ & $0,000^{\text {** }}$ \\
\hline FBZ & 46,4 a & $57,1 \mathrm{~b}$ & $60,8 \mathrm{c}$ & $0,000^{\text {*** }}$ \\
\hline
\end{tabular}

Ebenso gesicherte, deutliche Differenzen traten bei den Merkmalen der Schlachtleistung zwischen den Genotypen PP zu NP oder NN auf. Letztere zeigten gegenüber den zwei anderen Genotypen einen höheren Verfettungsgrad der Schlachtkörper, mit einem bis zu 0,32 cm stärkeren Seitenspeckmaß. Die Fleischfläche der NN-Tiere ist signifikant um $5,7 \mathrm{~cm}^{2}$ geringer als die der PP-Tiere. Das Fleisch-Fett-Verhältnis verschlechterte sich dadurch um 0,06 Einheiten. Der Fleischanteil und der Schinkenanteil verringerten sich bei den Genotypen NN nur um 2,7 \% bzw. 0,9\% und nicht in dem Ausmaß, wie durch den Rückgang der Fleischfläche zu erwarten gewesen wäre. Wird zu den Veränderungen der Schlachtleistungsmerkmale noch die signifkante Verbesserung der Schlachtkörperlänge von $1,6 \mathrm{~cm}$ bei NP- und von $2,2 \mathrm{~cm}$ bei NN-PI-Tieren zum Genotyp PP betrachtet, so zeigt sich auch bei der Rasse Piétrain durch die Selektion auf Streßresistenz eine Typumstellung wie bei der Rasse DL (WITTMANN et al., 1993).

Die größten Unterschiede ergaben sich für die Merkmale der Fleischbeschaffenheit, von denen nur die Differenzen im $\mathrm{pH}_{24}$-Schinken nicht abgesichert werden konnten. Sie verbesserten sich bei den Piétraintieren des Genotyps NN erheblich. So stieg der $\mathrm{pH}_{1}$-Kotelett um 0,65 und der $\mathrm{pH}_{1}$-Schinken um 0,71 Einheiten gegenüber der PPGruppe und um 0,19 und 0,30 Einheiten zur NP-Gruppe an. Auch die Unterschiede 
zwischen den NP- zu den PP-Tieren konnten abgesichert werden, obwohl die NP-Genotypen noch einen hohen Fleischanteil von $64,6 \%$ aufwiesen. Weniger ausgeprägt zeigten sich die Differenzen der $\mathrm{pH}_{24}$-Werte. Sie lagen im Kotelett um 0,07 Einheiten höher bei den NN- zu den PP-Tieren und um 0,06 zu den NP-Tieren. Der Unterschied zwischen der NP- zur PP-Genotypengruppe war nicht signifikant. Das gleiche Bild zeigten die Werte der Fleischhelligeit. Hier lagen die NN- und NP-Tiere mit bis zu 4,3 Punkten vor den PP-Tieren. Daraus resultierten auch die großen Differenzen in der Fleischbeschaffenheitszahl (FBZ). Die NN-Gruppe verbesserte sich gegenüber der NPund der PP-Gruppe um 3,7 bzw. 14,4 Punkte und die NP- zu den PP.-Tieren noch um 10,7 Punkte.

Die Verteilung der PP- und NP-Tiere nach den Fleischbeschaffenheitsmängeln PSE und DFD zeigt die Tabelle 3. Nur 28,4\% der PP-Tiere hatten $45 \mathrm{~min}$. p. m. eine einwandfreie Kotelettbeschaffenheit mit einem $\mathrm{pH}_{1}$ von $>5,80$, 27,6 \% lagen PSE-verdächtig zwischen 5,60 und 5,80 und 44,0\% wiesen mit $<5,60$ PSE-Fleisch auf. Bei den NP-Tieren kehrte sich die Verteilung um. 86,5\% zeigten eine normale Fleischbeschaffenheit im Rückenmuskel, 9,3\% hatten PSE-Verdacht und nur 3,6 \% PSEFleisch.

Tabelle 3

Verteilung der weiblichen PI-HB-Tiere in den MHS-Genotypen PP und NP nach Fleischbeschaffenheitsmängeln (Distribution of the female PI-HB-animals by meat quality and MHS-genotypes)

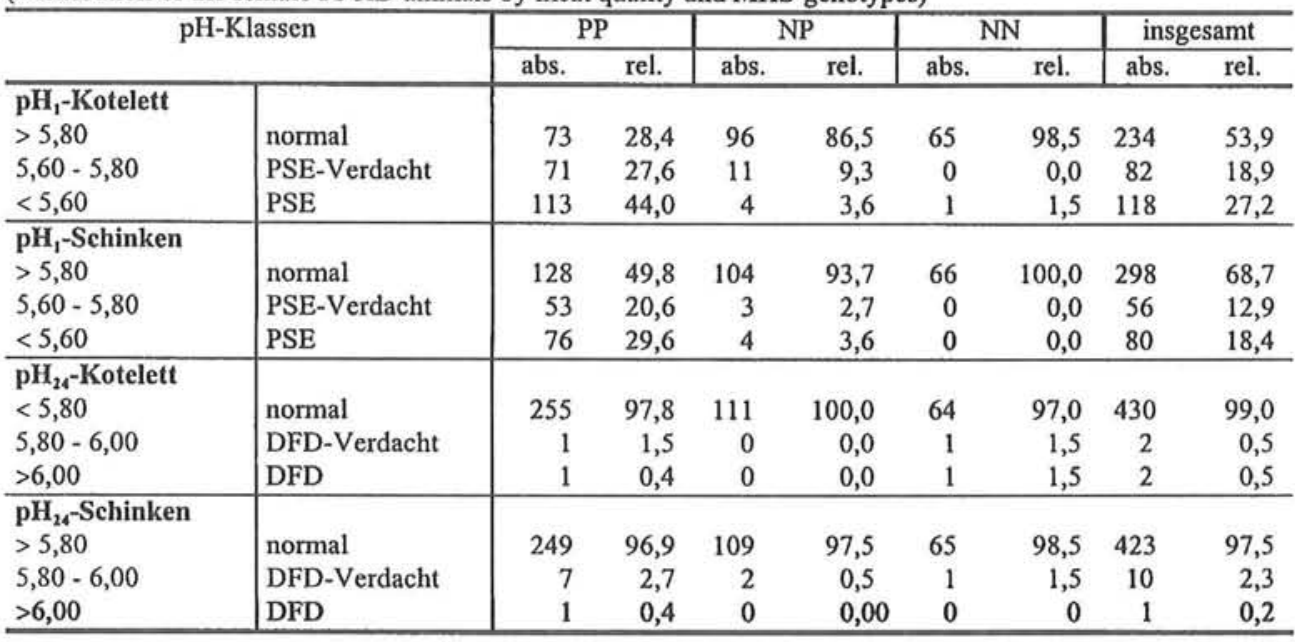

Von den NN-Tieren hatten $98,5 \%$ eine gute bis sehr gute Fleischbeschaffenheit und nur bei einem Tier wurde PSE-Fleisch im Kotelett beobachtet. Im Schinken entwikkelten die PP-Tiere mit 29,6 \% weniger PSE-Fleisch als im Kotelett, 20,6 \% lagen noch im Verdachtsbereich und 49,8\% im normalen Bereich. Eindeutig verbesserten sich bereits die NP-Tiere auf 93,7\% mit normaler Fleischbeschaffenheit, 2,7\% mit PSE-Verdacht und 3,6 \% mit PSE-Mangel. Für die NN-Tiere konnte zu $100 \%$ im Schinken eine gute Fleischbeschaffenheit nachgewiesen werden.

Nach den Abgrenzungen auf DFD-Eigenschaft im Rückenmuskel waren 97,8\% der 
PP-Tiere ohne Mangel $\left(\mathrm{pH}_{24}<5,80\right)$ und nur je 1 Tier zeigte Verdacht auf DFD- $\left(\mathrm{pH}_{24}\right.$ von 5,80 - 6,00) bzw. DFD-Fleisch $\left(\mathrm{pH}_{24}>6,00\right)$. Ein ähnliches Bild ergab die NNund die NP-Gruppe mit nur jeweils einem bzw. zwei DFD verdächtigen Tieren.

Die vorliegenden Ergebnisse bestätigen nicht eindeutig die früheren von PESCHKE et al. (1993). Als Ursache kann die in diesem Material breitere genetische Basis angesehen werden und die Tatsache, daß die Züchter bereits intensiv gegen den Leistungsabfall selektieren. Dies zeigt sich auch in Tabelle 4 anhand der BLUP-Zuchtwerte der lebenden Eber (Stand Sept. 1997) und ihrer Leistungsunterschiede nach MHS-Status. Auch hier bestätigt sich, daß NN-Eber eine bessere Zunahme gegenüber PP-Ebern aufweisen. Lediglich im Fleischanteil sind die NN-Eber im Naturalzuchtwert um $-1,61 \%$ schlechter als PP-Eber, was im absoluten Wert nach Tabelle $5-1,3 \%$ entspricht.

Tabelle 4

Unterschiede im Zuchtwert der lebenden PI-Eber nach MHS-Status (Differences in the breeding value of living PI-boars by MHS-status)

\begin{tabular}{cccc}
\hline Zuchtwert & PP & NN & Diff. \\
\hline Fleischanteil & $+0,58$ & $-0,93$ & $-1,61$ \\
FBZ & $-0,8$ & $+7,5$ & $+8,3$ \\
tägl. Zunahme & $-6,0$ & $+18,7$ & $+24,7$ \\
Gesamt & 105,2 & 131,1 & $+25,9$ \\
\hline
\end{tabular}

\section{Tabelle 5}

Unterschiede in den Nachkommenleistungen der lebenden PI-Eber nach MHS-Status (Differences in the performances of the offspring of living PI-boars by MHS-status)

\begin{tabular}{cccc}
\hline Merkmal & PP & NN & Diff. \\
\hline Fleischanteil & 66,1 & 64,8 & $-1,3$ \\
FBZ & 51,1 & 62,1 & $+11,0$ \\
tägl. Zunahme & 697,6 & 713,4 & $+15,8$ \\
pH, Kotelett $_{\text {Schlachtk.länge }}$ & 5,74 & 6,26 & $+0,52$ \\
\hline
\end{tabular}

\section{2}

PI x DL-Mastendprodukte

Von den 64 PI x DL-Einfachkreuzungen wurden 6 Tiere nach den Richtlinien als Ausfälle erfaßt, wobei 5 von ihnen den Genotyp NP aufwiesen. Die 58 Tiere mit vollständigen Leistungsdaten zur Auswertung verteilten sich auf 16 Kastraten (55,2 \%) und 13 weibliche $(44,8 \%)$ mit dem MHS-Status NN und $17(58,6 \%)$ männliche sowie 12 (41,4\%) weibliche mit dem MHS-Genotyp NP (Tab. 6).

\section{Tabelle 6}

Verteilung der PI x DL-Mastendprodukte nach MHS-Genstatus und Geschlecht $(n=58)$ (Distribution of the PI $x$ DL-animals by MHS-gene status and sex)

\begin{tabular}{|c|c|c|c|c|c|c|}
\hline \multirow{3}{*}{ MHS - Genotyp } & \multicolumn{4}{|c|}{ Geschlecht } & \multirow{2}{*}{\multicolumn{2}{|c|}{ gesamt }} \\
\hline & \multicolumn{2}{|c|}{ Kastraten } & \multicolumn{2}{|c|}{ weibl. Tiere } & & \\
\hline & $\mathbf{n}$ & $\%$ & $\mathrm{n}$ & $\%$ & $\mathbf{n}$ & $\%$ \\
\hline NN & 16 & 55,17 & 13 & 44,83 & 29 & 50,00 \\
\hline NP & 17 & 58,62 & 12 & 41,38 & 29 & 50,00 \\
\hline $\mathrm{NN}+\mathrm{NP}$ & 33 & 56,90 & 25 & 43,10 & 58 & 100,00 \\
\hline
\end{tabular}


Wie die Tabelle 7 verdeutlicht, konnten die Unterschiede der LSQ-Mittelwerte im Merkmal Zunahmen im Prüfungsabschnitt zwischen den Geschlechtern und den MHSGenotypen gesichert werden.

Tabelle 7

LSQ-Mittelwerte der PI x DL-Mastendprodukte nach MHS-Genotypen und Geschlecht $(n=58)$ (LSQ-means of the PI x DL-animals by MHS-gene status and sex)

\begin{tabular}{|c|c|c|c|c|c|c|}
\hline \multirow[b]{2}{*}{ Merkmal } & \multicolumn{3}{|c|}{ LSQ-Mittelwert Geschlecht } & \multicolumn{3}{|c|}{ LSQ-Mittelwert MHS-Genotyp } \\
\hline & Kastrat & weibl.Tier & $\mathrm{p}$ - Wert & NN & NP & $\mathrm{p}$ - Wert \\
\hline Zunahme i. Abschnitt & 852 & 774 & $0,029^{*}$ & 781 & 836 & $0,022^{*}$ \\
\hline Schlachtkörperlänge & 98,1 & 97,8 & 0,715 & 98,1 & 97,8 & 0,617 \\
\hline Rückenspeck x & 2,6 & 2,3 & $15,21^{* *}$ & 2,5 & 2,4 & 0,990 \\
\hline Seitenspeck & 3,2 & 2,2 & $0,000 * * *$ & 2,9 & 2,5 & $0,015^{*}$ \\
\hline Muskeldicke & 6,6 & 7,1 & $0,004^{* *}$ & 6,6 & 7,1 & $0,001 * *$ \\
\hline Fettfläche & 21,7 & 17,3 & $0,000^{* *}$ & 20,5 & 18,6 & $0,039^{*}$ \\
\hline Fleischfläche & 51,5 & 57,7 & $0,000^{* * *}$ & 52,3 & 56,7 & $0,004 * *$ \\
\hline Fleisch:Fett-Verhältnis & 0,43 & 0,30 & $0,000^{\text {से }}$ & 0,40 & 0,33 & $0,008^{* *}$ \\
\hline Fleischanteil (LPA) & 56,7 & 61,0 & $0,000^{*}$ & 57,8 & 59,9 & $0,005^{* * *}$ \\
\hline $\mathrm{pH}_{1}$-Kotelett & 6,44 & 6,21 & $7,49^{\text {***}}$ & 6,50 & 6,15 & $0,000^{\text {*****}}$ \\
\hline $\mathrm{pH}_{1}$-Schinken & 6,57 & 6,34 & $0,000^{* * * *}$ & 6,59 & 6,32 & $0,000^{* * *}$ \\
\hline $\mathrm{pH}_{24}$-Kotelett & 5,46 & 5,44 & 0,578 & 5,48 & 5,42 & 0,055 \\
\hline $\mathrm{pH}_{24}$-Schinken & 5,57 & 5,58 & 0,915 & 5,62 & 5,53 & $0,033^{*}$ \\
\hline Fleischhelligkeit & 68,3 & 70,6 & 0,173 & 71,2 & 67,8 & $0,042^{*}$ \\
\hline
\end{tabular}

In allen Schlachtleistungsmerkmalen traten, bis auf die Schlachtkörperlänge, zwischen den Geschlechtern ebenfalls gesicherte Unterschiede auf. In den Merkmalen Seitenspeckdicke, Muskeldicke, Fleischfläche, Fleisch-Fett-Verhältnis, Fleischanteil (LPA), Bauchpunkte, Muskelfleischanteil und Muskeldicke nach FOM unterschieden sich die MHS-Genotypen signifikant. Die Seitenspeckdicke erhöhte sich bei den NN PI x DL-Einfachkreuzungen um $0,4 \mathrm{~cm}$, mit einer um $3,7 \mathrm{~cm}^{2}$ verringerten Fleischfläche und in der Folge um 0,06 Punkten erhöhtem Fleisch-Fett-Verhältnis. Die Qualität des Bauches verschlechterte sich ebenfalls (1,1 Punkte), aber nicht so stark wie bei den DL- und PI-HB-Tieren. Nach Bonner Formel sank der Fleischanteil um 1,8\%, nach FOM etwas stärker um 2,0\% ab und lag damit zwischen den DL- $(0,8 \%$, WITTMANN et al., 1993 ) und PI-HB-Tieren (1,9\%). Obwohl sich die Schlachtkörperlänge nicht signifikant verändert hatte, deutet die stärker abnehmende Fleischfläche als der Fleischanteil der streßresistenten Tiere auf eine leichte Typumstellung analog zu den DL-Kastraten und PI-HB-Tieren hin.

Sowohl bei den Geschlechtern als auch den MHS-Genotypen unterschieden sich die $\mathrm{pH}_{1}$-Werte im Schinken und Kotelett signifikant. Die PI x DL-Tiere des Genotyps NN verbesserten sich um 0,27 bzw. 0,35 Punkte im Schinken bzw. Kotelett. Auch im $\mathrm{pH}_{24^{-}}$ Schinken zeigten sie sich um 0,09 Punkte den NP-Tieren überlegen. Die Fleischhelligkeit der NN PI x DL-Tiere erhöhte sich um 3,4 Punkte, der Unterschied war jedoch statistisch nicht signifikant, im Gegensatz zu der Verbesserung von 2,5 Punkten bei PIHB-Tieren und 6,0 bei DL-Kastraten.

Die Aussage der pH-Werte spiegelte sich in der Verteilung der PI x DL-Tiere nach PSE- und DFD-Fleischbeschaffenheitsklassen wider (Tab. 8). In der NN-Genotypengruppe trat kein Schlachtkörper mit PSE-Eigenschaften auf und auch die NP-Tiere 
zeigten mit 93,1 \% einen sehr hohen Anteil an guter Fleischbeschaffenheit, bei nur 6,9 $\%$ mit PSE-Mangel. Alle NP-Genotypen lagen beim $\mathrm{pH}_{24}$-Kotelett im normalen Bereich von $<5,80$, ebenso 96,5\% der NN-Tiere, von denen nur eines DFD-Verdacht aufwies.

Tabelle 8

Verteilung der PI x DL-Mastendprodukte in den MHS-Genotypen NN und NP nach Fleischbeschaffenheitsmängeln (Distribution of the PI x DL-animals by meat quality and MHS-genotypes)

\begin{tabular}{|c|c|c|c|c|c|c|}
\hline \multirow{3}{*}{$\begin{array}{l}\text { Fleischbeschaffen- } \\
\text { heitsgruppe }\end{array}$} & \multicolumn{4}{|c|}{ MHS - Genotyp } & \multirow{2}{*}{\multicolumn{2}{|c|}{ gesamt }} \\
\hline & \multicolumn{2}{|c|}{$\mathrm{NN}$} & \multicolumn{2}{|c|}{ NP } & & \\
\hline & n & $\%$ & $\mathbf{n}$ & $\%$ & $\mathrm{n}$ & $\%$ \\
\hline $\mathrm{pH}_{1}$-Kotelett & & & & & & \\
\hline $\mathrm{PSE}<5,60$ & 0 & 0,0 & 2 & 6,9 & 2 & 3,5 \\
\hline PSE-Verdacht $5,60-5,80$ & - & - & - & - & - & - \\
\hline Normal $>5,80$ & 29 & 100,0 & 27 & 93,1 & 56 & 96,5 \\
\hline $\begin{array}{l}\mathbf{p H}_{24} \text {-Kotelett } \\
\text { Normal }<5,80\end{array}$ & 28 & 96.5 & 29 & 100.0 & 57 & 98.3 \\
\hline DFD-Verdacht $5,80-6,00$ & 1 & 3,5 & 0 & 0,0 & 1 & 1,7 \\
\hline $\mathrm{DFD}>6,00$ & - & - & - & - & - & - \\
\hline
\end{tabular}

4. Schlußfolgerungen

Die vorliegende Untersuchung bestätigt nur teilweise die früheren Befunde (WITTMANN et al., 1993; PESCHKE et al., 1993), daß reinerbig streßresistente Tiere in der Mastleistung unterlegen sind. Insbesondere das jüngere Material der PietrainReinzuchttiere zeigte, daß streßresistente Tiere auch überlegene Mastleistungen haben können.

In den Schlachtkörpermerkmalen bestätigten sich die aufgrund der Ergebnisse bei der Rasse DL (WITTMANN et al., 1993) vorliegenden Erwartungen. Streßresistente Tiere weisen erhöhte Speck- und verringerte Fleischmaße auf. Der Magerfleischanteil verringert sich von PP über NP zu NN, wobei jedoch die Differenz von PP zu NN deutlich größer ist als der Unterschied zwischen misch- und reinerbig streßresistenten Tieren.

Im Hinblick auf die Fleischbeschaffenheit ist die größte positive Veränderung beim Übergang von PP zu NP zu beobachten. Dennoch war sowohl in den $\mathrm{pH}_{1}$-Werten, als auch in der FBZ noch eine signifikante Verbesserung der NN- gegenüber den NP-Genotypen festzustellen. Die Einteilung in Fleischqualitätsklassen bestätigt diese Befunde.

Die untersuchten Endprodukte stammten von den streßresistenten Pietrainebern der ersten Generation ab. Diese zeigten noch eine deutliche Unterlegenheit in der Mastleistung (PESCHKE et al., 1993). Die Ergebnisse der derzeit aktuellen streßresistenten Pietraineber in Bayern beweisen jedoch, daß sich im Vergleich zur damaligen Untersuchung die Unterlegenheit im Fleischanteil verringert hat und daß im Bereich der täglichen Zunahmen sogar eine deutliche Überlegenheit der NN-Eber festzustellen ist. Geht man davon aus, daß sich auch in Zukunft der Trend zu einer rückläufigen Bezahlung extremer Magerfleischanteile in Verbindung mit steigenden Schlachtgewichten fortsetzen wird, dann entspricht der NN-Eber diesen Anforderungen am besten. 
Eine Streßsanierung der Vaterrasse Piétrain zur Verbesserung der Fleischqualität und Gesamtwirtschaftlichkeit ist daher empfehlenswert.

\section{Literatur}

BIEDERMANN, G.; PESCHKE, w.; WITTMANN, W.; BRANDT, C.:

Stand der Zucht-, Mast- u. Schlachtleistung von Piétrain-Schweinen unterschiedlichen MHS-Genotyps aus zwei Zuchtherden. Arch. Tierz., Dummerstorf 40 (1997) 6, 535-547

DOVC, P.; BIRKENMAIER, S.; FÖRSTER, M.:

Der Münchner MHS-Test im Praxiseinsatz. Vortragstagung der DGfZ/GfT (1992), Weihenstephan

GLODEK, P.:

Brauchen wir uberhaupt noch streßanfallige Schweinerassen in der deutschen Schweineproduktion? DGfZ-Schriftenreihe H. 4 (1996), 191-200

GROENEVELD, E.:

PEST User Manual, Version 3.1 (1993), Inst. für Tierzucht und Tierverhalten der FAL, Mariensee

PESCHKE, W.; FÖRSTER, M.; ODEBRECHT, S.; DOVC, P.; BEHRINGER, J.:

Leistungsunterschiede zwischen MHS reinerbig positiven und mischerbigen Piétrainschweinen in der Stationsprüfung (1993), Vortragstagung der DGfZ/GfT in Göttingen

SCHMUTZ, M.:

Multivariate Schătzung von Populationsparametern fur Merkmale aus der Stations- und Feldprüfung der bayerischen Schweine-Herdbuchzucht in Reinzucht und Kreuzung. Univ. Kiel, Diss., 1995

THOLEN, E.:

Genitgt ein mischerbiges streBfreies Endprodukt? DGfZ-Schriftenreihe H. 4 (1996), 181-190

WITTMANN, W.; HOJNY, J.; SCHMID, D.-O.; KRAUS, H.; HAUSER, M.; BLENDL, M.-H.:

Selektion homozygot streßresistenter Linien bei DL-Schweinen mittels Markergenen. Arch. Tierz., Dummerstorf 35 (1992), 9-127

WITTMANN, W.; PESCHKE, W.; LITTMANN, E.; BEHRINGER, S.; BIRKENMAIER,S.; DOVC, P.; HAUSER, M.:

Mast- und Schlachtleistung von DL-Kastraten in Abbhängigkeit vom MHS-Genotyp. Züchtungskunde, Stuttgart 65 (1993), 197-205

Eingegangen: 13.05 .1998

Akzeptiert: 04.09.1998

Anschriften der Verfasser

Dr. agr. WERNER WITTMANN, Dr. agr. KAY-UWE GÖTZ, LLD WALTER PESCHKE, MAXIMILIAN HAUSER

Bayerische Landesanstalt für Tierzucht, Grub

Prof.-Dürrwaechter-Platz 1

D-85586 Poing

Dr. agr. JOHANN-PETER LINDNER

Lehr- und Versuchsanstalt für Tierhaltung Schwarzenau

D-97359 Schwarzach 


\section{Berichtigung}

Im Beitrag „Der Einfluß des MHS-Streßgens auf die Fleischleistung und Fleischbeschaffenheit der Nachkommen von Pietrain- und (Pietrain * Hampshire)Kreuzungsebern" von GÖDEKE et al. aus dem Heft 5 unserer Zeitschrift (Band 41, 1998, Seiten 433-446) ist die Tabelle 6 im unteren Tabellenteil zu korrigieren, wofür wir um Verständnis bitten.

Die Tabelle 6 wird wie folgt geändert:

\section{Tabelle 6}

Verschiedene Wirtschaftlichkeitsrechnungen ohne und mit PSE-Abzug (15 DM/Tier) (Various economical calculations without and with PSE-deduction (15 DM/animal))

\begin{tabular}{|c|c|c|c|c|c|c|c|}
\hline \multirow[t]{2}{*}{ Kriterium } & \multirow{2}{*}{$\begin{array}{c}\text { Gesamt- } \\
\text { mittel }\end{array}$} & \multicolumn{4}{|c|}{ Abweichungen der Gruppen } & \multicolumn{2}{|c|}{ Differenz } \\
\hline & & PI (NN) & PI (NP) & $\mathrm{PI}^{*} \mathrm{HA}(\mathrm{NN})$ & $\mathrm{PI}^{*} \mathrm{HA}(\mathrm{NP})$ & PI-PI*HA & NN-NP \\
\hline Masttage & 129,26 & $+0,89$ & $+1,16$ & $-0,35$ & $-1,70$ & $+2,05$ & $+0,54$ \\
\hline Futteraufnahme & 288,15 & $+2,42$ & $+3,10$ & $-0,48$ & $-5,04$ & $+5,52$ & $+1,94$ \\
\hline FOM-Fleischanteil $\%$ & 56,70 & $-0,07$ & $+0,38$ & $-0,48$ & $+0,17$ & $+0,32$ & $-0,56$ \\
\hline FOM-Speckmaß $\mathrm{mm}$ & 15,72 & $+0,16$ & $-0,04$ & $+0,20$ & $-0,32$ & $+0,12$ & $+0,36$ \\
\hline FOM-Fleischmaß $\mathrm{mm}$ & 62,86 & $+0,04$ & $+1,66$ & $-1,49$ & $-0,20$ & $+1,70$ & $-1,46$ \\
\hline pH45 (Kotelett) $<5,8 \%$ & 11,0 & $-3,2$ & $+13,1$ & $-8,5$ & $-1,5$ & $+10,0$ & $-11,7$ \\
\hline $\mathrm{pH} 45$ (Kotelett) $<6,0 \%$ & 34,9 & $-9,6$ & $+25,1$ & $-17,8$ & $+2,3$ & $+15,5$ & $-27,4$ \\
\hline Futterkosten & 112,38 & $+0,94$ & $+1,21$ & $-0,19$ & $-1,97$ & $+2,16$ & $+0,76$ \\
\hline Erlös nach NW-Not. DM/Tier & 253,50 & $-0,42$ & $+1,39$ & $-0,32$ & $-0,66$ & $+0,98$ & $-0,74$ \\
\hline BEG-Prämie & 15,46 & $-1,33$ & $+0,38$ & $-0,42$ & $+1,36$ & $-0,95$ & $-1,75$ \\
\hline Überschuß DM/Tier & & & & & & & \\
\hline NW o. Fleischbeschaffenheit & & $-1,57$ & $+1,41$ & $-1,16$ & $+1,33$ & $-0,20$ & $-2,74$ \\
\hline m. PSE $(\mathrm{pH}<5,8)$ & & $-1,09$ & $-0,58$ & $+0,12$ & $+1,55$ & $-1,70$ & $-0,99$ \\
\hline m. PSE $(\mathrm{pH}<6,0)$ & & $-0,13$ & $-2,36$ & $+1,52$ & $+0,98$ & $-2,53$ & $+1,37$ \\
\hline BEG o. Fleischbeschaffenheit & & $-2,99$ & $+1,77$ & $-1,63$ & $+2,84$ & $-1,28$ & $-4,59$ \\
\hline m. PSE $(\mathrm{pH}<5,8)$ & & $-2,51$ & $-0,21$ & $-0,36$ & $+3,07$ & $-2,78$ & $-2,84$ \\
\hline m. PSE $(\mathrm{pH}<6,0)$ & & $-1,55$ & $-2,00$ & $+1,04$ & $+2,50$ & $-3,61$ & $-0,48$ \\
\hline
\end{tabular}

